Pubic reponing butden for inis collectlon of information is estimated to avera

maintalning the da:a needed. and completing and revlewing the collectlon of information Send comments regarding this burden estimate or any other aspect of inls collection of information incluging

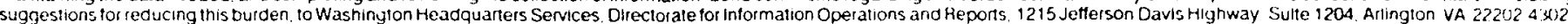
and to the Oflice of Management and Budget Paperwork Reductlon Piolect (0704-0188). Washington DC, 20503

\begin{tabular}{|l|r|}
\hline I AGENCY USE ONLY (LCove Diank) & $\begin{array}{r}\text { REFORI DATE } \\
\text { May } 1991\end{array}$ \\
\hline
\end{tabular}

4 TITLE AND SUBTITLE

GEOMETRIC CHARACTERIZATION OF EIGENVALUES OF COVARIANCE MATRIX FOR TWO-SOURCE ARRAY PROCESSING 6 AUTHORIS)

S. 1. Chou

7 PERFORMING ORGANIZATION NAME(S) AND AMDRESS(ES)

Naval Ocean Systems Center

San Diego, CA $92152-5000$

9 SPONSORING/MONITORING AGENCY NAME(S) AND ADDRESSIES

Naval Ocean Systeins Center

Block Programs

San Diego, CA 92152-5000

11 SUPPLEMENTARY NOTES

$\checkmark$

$+\cdots, \cdots$

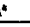

$\frac{7}{3}$

ran

.

10 SPONSORING/MONITORI

O SPONSORING/MONITORING AGENCY REPORT NUWBER

WU: $1(S \times B 200$

PE: 0602111N

Professional Paper

5 FUNDING NUMBEFAS

8. PERFORMING ORGA S
REPORT NUMBER

$\because+\ldots, 1 ;:$

$\ldots \quad \cdots \neq 1$

HAER $\quad \cdots, \ldots$

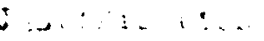

-... - - - -

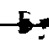

$1:$

\&.

Tas, onjice.

12a DISTRIBUTION/AVAILABILITY STATEMENT

12b DISTRIBUTION CODE

$2+1: 4$

Approved for public release; distribution is unlimited.

13 ABSTRACT (Maximum 200 words)

For a two-source array processing scenario, normalized eigenvalues' expressions $\lambda_{1}$ and $\lambda_{2}$ are reduced to forms depending only on a real triplet: phase-dependent, variable $\xi$, phase-independent variable $\eta$, and power ratio $\frac{\pi_{1}}{\pi_{2}} \cdot(\xi, \eta)$
is confined to an isosceles-like region. We characterize

- this isosceles-like region and the many-to-one mapping from the Cartesian product of the temporal an spatial correlation unit-disks onto this region,

- the behavior of the eigenvalues and their ratio as functions of the real triplet with respect to array processing, and

- a characterization of Speiser's eigenvalue bounds specialized to the two source scenario.

Published in ICASSP91 Proceedings, 14 May 1991.

\begin{tabular}{|c|c|c|c|}
\hline \multicolumn{3}{|l|}{14 SUdirnt TFRMS } & 15 NUMBER OF PAGES \\
\hline \multirow{2}{*}{$\begin{array}{l}\text { tracker/coorrelator } \\
\text { dim target }\end{array}$} & \multirow{2}{*}{\multicolumn{2}{|c|}{$\begin{array}{l}\text { ultra wideband radar } \\
\text { HFDF }\end{array}$}} & \\
\hline & & & 16 PRICE CODE \\
\hline $\begin{array}{l}17 \text { SECURITY CLASSIFICATION } \\
\text { OF REPORT }\end{array}$ & $\begin{array}{l}\text { 18 SECURIYY CLASSIFICATION } \\
\text { OF THIS PAGE }\end{array}$ & $\begin{array}{l}19 \text { SECURITY CLASSIFICATION } \\
\text { OF ABSTRACT }\end{array}$ & 20 LIMITATION OF ABSTRACT \\
\hline UNCLASSIFIED & UNCIAASSIFIED & UNCLASSIFIED & SAME AS REPORT \\
\hline
\end{tabular}




\title{
$\because$ GEOMETRIC CHARACTERIZATION OF EIGENVALUES OF COVARIANCE MATRIX FOR TWO-SOURCE ARRAY PROCESSING
}

\author{
S.I. ('hon \\ Navai, OClin Systrims Center \\ SAN DiEgo, CA 92152-5000
}

\section{Abstract}

For a two-source array processing scenario, normalized eigenvalues' expressions $\lambda_{1}$ and $\lambda_{2}$ are reduced to forms depending only on a real triplet: phase-dependent variable $\xi$, phase-independent variable $\eta$, and power ratio $\frac{\pi_{1}}{\pi_{2}} \cdot(\xi, \eta)$ is confined to an isosceles-like region. We characterize

- this isosceles-like region and the many-to-one mapping from the Cartesian product of the temporal and spatial correlation unit-disks onto this region,

- the behavior of the eigenvalues and their ratio as functions of the real triplet with respect to array processing, and

- a characterization of Speiser's eigenvalue bounds specialized to the two source scenario.

\section{Introduction}

The eigenvalues play a central role in parametric signal subspace fitting methods, in many versions of nonparametric subspace beamforming, and in applying the rank-reduction principle to subspace array processing.

Very scanty discussion of the eigenvalue expressions not restricted to the simple uncorrelated arrivals was given in [1] for two-source array processing. The eigenvalues are solutions of a quadratic equation but they depend on 6 real parameters, i.c., with 4 from the spatial and temporal complex corrclations and 2 from the power levels of the arrivals.

A companion paper[2] contains minimum mathematical development but concentrates on the overview of the problem, graphic display, physical interpretation of results. This paper provides some details on which the companion paper is based and then characterize Speiser's eigenvalue bounds[3] specialized to the two source scenario.

We give special treatment for equal power arrivals because of its many unique characteristics and its importance in real applications.

The analytical treathent begins by reviewing the expressions of the noise-free eigenvalues of the guadratic characteristic equations of the non-Hermitian product of the temporal and spatial correlation matrix given in IIudson's text $[1, \mathrm{pP} .52-55]$. The eigenvalues are normalized with respect to the product of the number of sensors and the sensor level power of the weaker source $\pi_{2}$, i.c., $\pi_{1} \geq \pi_{2}$. The normalized large and small eigenvalues expressions $\lambda_{1}$ and $\lambda_{2}$ are reduced to forms depending only ont the real t.riplet $\left(\xi, \eta, \frac{\pi_{1}}{\pi_{2}}\right)$. Here, $\frac{\pi_{1}}{\pi_{2}}$ is the power ratio between the strong to weak sources at the sensor level. The real pair $(\xi, \eta)$ are defined in terms of the normalized temporal and spatial coefficients $\rho$ and $\phi$ respectively with each constrained to a unit-clisk through

$$
\begin{gathered}
\eta=\left(1-|\phi|^{2}\right)\left(1-|\rho|^{2}\right), \\
\xi=\operatorname{Re}\left(\rho \phi^{*}\right)=|\rho||\phi| \cos (\arg \rho-\arg \phi) .
\end{gathered}
$$

Using our notation, the normalized eigenvalues assume the following form

$$
\begin{aligned}
\left(\begin{array}{l}
\lambda_{1} \\
\lambda_{2}
\end{array}\right) & =\frac{1}{2}\left[\frac{\pi_{1}}{\pi_{2}}+1+\left(\frac{\pi_{1}}{\pi_{2}}\right)^{\frac{1}{2}} 2 \xi\right] \\
& \times\left[1 \pm \sqrt{1-\frac{4\left(\frac{\pi_{1}}{\pi_{3}}\right) \eta}{\left[\frac{\pi_{1}}{\pi_{2}}+1+\left(\frac{\pi_{2}}{\pi_{2}}\right)^{\frac{1}{2}} 2 \xi\right]^{2}}}\right] .
\end{aligned}
$$

While the discussions of eigenvalues in this report are applicable to generic steering vectors, we call $\eta$ the phaseindependent variable and $\xi$ the phase-dependent variable for convenience even though such names were motivated by the special case of plane waves impinging onto pairwise symmetric arrays.

\section{An Isosceles Right-Triangle-Like Region}

The phase-independent variable $\eta$ depends only on the magnitude of $\rho$ and $\phi$. The phase-dependent variable $\xi$ depends also on the angular positions of $\rho$ and $\phi$. The mapping from the complex pair $(\rho, \phi)$ to the real pair $(\xi, \eta)$ is many to one. The range of this mapping is an isosceles right-trianglelike region bounded on its left and right hy two symmetric parabolas, $\eta=(\xi \pm 1)^{2}$, and down below by a straight baseline, $\eta=0$. We note the zero slope of each parabola where it meets the straight line and the $90^{\circ}$ angle that the two parabolas intersect each other.

We will see later that among the three parameters $\left(\xi, \eta, \pi_{1} / \pi_{2}\right)$ appearing in the eigenvalues' expressions, $\eta$ is the most important one.

The two eigenvalues are equal if and only if what is inside the radical of the eigenvalue equation vanishes. It can be shown that for this to be true we must have $\pi_{1} / \pi_{2}=1$. and llant $\xi$ nuel $y$ unist be on the left parabola of the isosceles-like region. The common eigenvalue value they share is

$$
\lambda_{i}\left(\xi,(1+\xi)^{2}\right)=(1+\xi)=\sqrt{\eta} .
$$

\section{Characterizing Eigenvalues and their Ratio}

We characterize the belavior of the eigenvalues and their ratio $\frac{\lambda_{1}}{\lambda_{2}}$ as functions of the real triplet $\left(\xi, \eta, \frac{\pi_{1}}{\pi_{2}}\right)$. It is useful to discuss the expressions for the eigenvalue and their ratio $\frac{\lambda_{1}}{\lambda_{2}}$ for the special cases at the apex, the baseline, and the vertical axis. But instead, we characterize the snecial 
case of equal-strength arrivals and then the general case will be touched upon only lightly at the end.

\section{Eigenvalues}

The small eigenvalue will be seen to diminish qualitatively and quantitulively for two-arrival scenurios increasingly stressed with high temporal and/or spatial correlations. The special case of equal strength $\frac{\pi_{1}}{\pi_{2}}=0 \mathrm{~dB}$ arrivals, also important in low-angle radar tracking, shares many rich structures of general $\frac{\pi_{1}}{\pi_{3}}$. It has several additional unique reatures for signal cigcnvalues' ratio $\frac{\lambda_{1}}{\lambda_{2}}$ importunt in arrny processing.

The special case of equal strength $\frac{\pi_{2}}{\pi_{2}}=0 \mathrm{~dB}$ arrivals shares the following common features of general $\frac{\pi_{1}}{\pi_{3}}$ power ratios: straight line contours for constant cigenvalue $\lambda$ 's, hyperbciic $\lambda$ slices for constant phase-independent variable $\eta$ 's, and parabolic $\lambda$ slices for constant phase-dependent variable $\xi$ 's. Ilowever, we caution that unless special effort is made in plotting results, the two displayed surfaces or curves may not always meet at the supposed places. This is because the boundary curves defining parabolas in the $\xi$ and $\eta$ plane, in general, do not pass through the grid points used in the $\xi$ and $\eta$ plane.

As $\eta$ approaches 0 , the small cigenvalue $\lambda_{2}$ and the eigenvalue ratio $\frac{\lambda_{1}}{\lambda_{2}}$ tend toward - and $+\infty$ di3, respectively. Similarly, ns $\eta$ nppronches 0 for the cquipower case, $\frac{\pi_{1}}{\pi_{2}}=0$ dI3, both the upper $\lambda_{1}$ and lower $\lambda_{2}$ hyperholas tend toward their asymptotes intersceting at $-\infty$ dIB.

One can use plots to give some inclication of the $\xi$ dependent nature of the eigenvalues. But it is possible to analyze the same phenomenon for the most important area. As the $\eta=0$ baseline is where $\lambda_{2}=0$, we have more quantitative information about the $\xi$-dependent or $\xi$-independent nature of $\lambda_{2}$ in this neighborhood for given values of $\frac{\pi_{1}}{\pi_{3}}$ because of the following characterization. For given $\xi$ and $\frac{\pi_{1}}{\pi_{2}}=1$, as for all other values, the stress of a directionfinding scenario is highest when the baseline $\eta$ is approached We are interested in the local behavior at the stressful region about $\eta=0$. In this region, the large eigenvalue $\lambda_{1}$ will not change much in terms of its order of magnitude. This is not the case for the small eigenvalue $\lambda_{2}$, as it is the difference of two nearly equal numbers. The local first-order approximations to $\lambda_{1}$ and $\lambda_{2}$ near $\eta=0$ are therefore

$$
\begin{gathered}
\lambda_{1}(\xi, \eta) \approx \lambda_{1}(\xi, 0)=2(1+\xi), \\
\lambda_{2}(\xi, \eta) \approx \frac{\eta}{2(1+\xi)} .
\end{gathered}
$$

Near $\eta=0$ and at the three particular $\xi$ values, we have for $\frac{x_{1}}{\pi_{1}}=1$

$$
\lambda_{2}(-1, \eta) \approx \infty \eta, \quad \lambda_{2}(0, \eta) \approx \frac{\eta}{2}, \quad \lambda_{2}(1, \eta) \approx \frac{\eta}{4}
$$

This indicate the significant $\xi$-dependence of $\lambda_{2}$.

Near $\eta=0$ and for large $\frac{\pi_{1}}{\pi_{2}}$, we have instead

$$
\begin{gathered}
\lambda_{1}(\xi, \eta) \approx \lambda_{1}(\xi, 0)=\frac{\pi_{1}}{\pi_{2}}+1+\sqrt{\frac{\pi_{1}}{\pi_{2}}} 2 \xi, \\
\lambda_{2}(\xi, \eta) \approx \eta
\end{gathered}
$$

i.e., essentially $\xi$-independent.
Eigenvalue: Ralio

We need to know the shape of the contours of the cigenvalue ratio $\frac{\lambda_{1}}{\lambda_{2}}=l$, i.e.,

$$
\frac{\lambda_{1}}{\lambda_{2}}=\frac{1+\sqrt{ }}{1-\sqrt{r}}=l,
$$

where $\sqrt{ }$ assumes the expression from equation 1 . This is equivalent to

$$
\frac{1\left(\frac{\pi_{1}}{\pi_{2}}\right) \eta}{\left[\frac{\pi_{1}}{\pi_{2}}+1+\left(\frac{\pi_{1}}{\pi_{2}}\right)^{\frac{1}{2}} 2 \xi\right]^{2}}=\frac{1 l}{(l+1)^{2}} .
$$

For the special case of equal strength $\frac{\pi_{1}}{\pi_{3}}=0 \mathrm{~dB}$, the contours of the eigenvalue ratio $\hat{\lambda}_{2}=l$ form a oneparameter family of parabolas having their vertices colocated at $(\xi, \eta)=(-1,0)$, i.e., at the lower left corner of the isosceles-like triangle and their common tangent the baseline $\eta=0.1$ This family of parabolas includes the left boundary of the isosceles-like triangle and the bascline. Because the contours of the eigenvalue ratio $\frac{\lambda_{1}}{\lambda_{2}}=l$ pass through the lower left corner of the isosceles-like triangle, this corner is a point of discontinuity for the eigenvalue ratio $\frac{\lambda_{1}}{\lambda_{2}}$ for the special case of equal strength $\frac{\pi_{2}}{\pi_{2}}=0 \mathrm{~dB}$. For example, the two eigenvalues are equal over the left parabolic houndary of the isosceles right-triangle-like region. Furthermore, the two equally dominant eigenvalues go down to zero jointly so thai the comdition mumber stays as mity throughout. 'l hat is, the condition numbers are the lowest possible there. However, both eigenvalues tend to zero as $\eta$ tends to zero, i.e., the baseline of the region. The limit of $\frac{\lambda_{1}}{\lambda_{3}}$ approaching from the bascline of the isosceles region is $\infty_{2}$, while that approaching from the left houndary curve of the isosceles region is 0 .

For the equi-powered case, the destructive interference scenario point is at the left apex of the isosceles region. Both cigenvalues are zero and the eigenvalue ratio $\frac{\lambda_{1}}{\lambda_{2}}$ at this point is of the $0 / 0$ form. This point corresponds to the total cancellation of signals at the sensor element level. The total cancellation happens not only at the center of the array but at every sensor. The two steering vectors coincide and the temporal waveforms negate each other completely. Consider two strong sources behaving this way. As the power is measured by turning off one source at a time, so the equal power are considered as high and CRI,B and asymptotic results apply when noise is taken into consideration.

We focus attention to the condition number's behavior over the vertical axis $\xi=0$ of the isosceles region. For the mpecial case of cqunl slrengl.h $\pi_{1}=0$ dl\}, there is an extra $6 \mathrm{~d}$ B for signal eigenvalues' ratio $\frac{\lambda_{1}}{\lambda_{2}}$ over the vertical axis $\xi=0$ accounting for the presence of an infinite slope of $\lambda$ 's with respect to $\eta$ at the apex for the equal-power arrival case. This is reflected in the following two approximations.

When $\frac{\pi_{1}}{\pi_{2}} \gg 1$, we first approximate the radical expression as

$$
\sqrt{1-\frac{1\left(\frac{\pi_{1}}{\pi_{2}}\right) \eta}{\left(\frac{\pi_{1}}{\pi_{2}}+1\right)^{2}}} \approx \sqrt{1-\frac{4 \eta}{\frac{\pi_{1}}{\pi_{2}}}} \approx 1-\frac{2 \eta}{\frac{\pi_{1}}{\pi_{2}}} .
$$

${ }^{1}$ For the general case, i.e., $\frac{\pi_{1}}{\pi_{2}}>1$, this common vertex is moved to the left from $(-1,0)$ and is now outside of the isosceles-like region. 


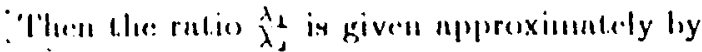

$$
\frac{\lambda_{1}}{\lambda_{2}}=\frac{1+\sqrt{ }}{1-\sqrt{2}} \approx \frac{2-\frac{\frac{2 \eta}{2}}{\frac{1}{\pi_{2}}}}{\frac{\frac{2 \eta}{2}}{\frac{1}{2}}}=\frac{\frac{\pi_{1}}{\pi_{2}}}{\eta}-1 \approx \frac{\pi_{1}}{\pi_{2}} / \eta
$$

That means the stress from $\eta$ and $\frac{\pi_{1}}{\pi_{2}}$ is multiplicative (divisive) in the direct scale. In dB scale, this is additive, i.e., when $\frac{\pi_{1}}{\pi_{2}}>10 \mathrm{~dB}$, we have

$$
\frac{\lambda_{1}}{\lambda_{2}}(\text { ind d B }) \approx\left(\frac{\pi_{1}}{\pi_{2}}\right)(\text { in d IB })-\eta(\text { in } \text { (IB) }
$$

When $\frac{\pi_{1}}{\pi_{3}} \approx 1$, we have

$\frac{\lambda_{1}}{\lambda_{2}}=\frac{1+\sqrt{1-\eta}}{1-\sqrt{1-\eta}}=\frac{(1+\sqrt{1-\eta})^{2}}{1-(1-\eta)}=\frac{2-\eta+2 \sqrt{1-\eta}}{\eta} \approx \frac{4}{\eta}$

We used $\eta \ll 1$ in the last step. When $\frac{\pi_{1}}{\pi_{2}} \approx 0$ dB and $\eta$ is less than 0.10 (or -10 (II), we have

$$
\frac{\lambda_{1}}{\lambda_{2}}(\text { in } d B) \approx-\eta(\text { in } d B)+6 .
$$

The variation of signal eigenvalue ratio $\frac{\lambda_{2}}{\lambda_{2}}$ across the phase-dependent variable $\xi$ is not significant. For the special case of equal strength $\frac{\pi_{2}}{\pi_{2}}=0 \mathrm{~dB}$, there is a 4-time or $6 \mathrm{~dB}$ increase on the eigenvalue ratio $\frac{\lambda_{1}}{\lambda_{2}}$ for highly correlated cquipower arrivals both temporally and spatially from changing the phase of the source correlation or the angle difference between the two unit-clisk vectors from $90^{\circ}$ to $\left(0^{\circ}\right.$.

As power ratio $\frac{\pi_{1}}{\pi_{2}}$ increases from unit value, the variation of the large eigenvalue $\lambda_{1}$ is essentially along the direction of the horizontal phase-dependent variable $\xi$. The smaller cigenvalue $\lambda_{2}$ is indeperident of both the horizontal phase-dependent variable $\xi$ and $\frac{\pi_{1}}{\pi_{2}}$ near the bascline for large $\frac{\pi_{1}}{\pi_{2}}$ but is a strong function of the vertical phaseindependent variaile $\eta$. The effect of $\frac{\pi_{1}}{\pi_{2}}$ is essentially only felt by the large eigenvalue which is not sensitive to whether the scenario's $(\xi, \eta)$ coordinate is close to the baseline, i.e., whether the arrivals are correlated or close to each other as far as its order of magnitude is concerned.

We have used these results for assessment of scenarios in [4] from which this paper and [2] are excerpted.

\section{Specializing Speiser's Eigenvalue Bounds to Two Sources}

As we have the exact expressions of the eigenvalues for the two-source scenario, we can examine quantitatively some general multisource results by specializing them to this case. For example, we consider the singular value bounds derived by Speiser and Arnold[3] for the product of two square matrices. Specialized to the two-source scenario and in terms of eigenvalues in our context, his expression can be written as

$$
\sigma_{2}^{2}(P) \sigma_{2}^{2}(\Psi) \leq \lambda_{2} \leq \min \left[\sigma_{2}^{2}(P) \sigma_{1}^{2}(\Psi), \sigma_{1}^{2}(P) \sigma_{2}^{2}(\Psi)\right],
$$

where

$$
P=\left[\begin{array}{cc}
\frac{\pi_{1}}{\pi_{2}} & \sqrt{\frac{x_{1}}{\pi_{2}} \rho} \\
\sqrt{\frac{\pi_{1}}{\pi_{2}}} \rho^{*} & 1
\end{array}\right], \quad \Psi=\left[\begin{array}{cc}
1 & \phi \\
\phi^{*} & 1
\end{array}\right],
$$

nut $\sigma_{i}^{2}(\Psi)$ is the i-th lurgest cigensalue of the llemilian matrix $\Psi$, and $\lambda_{i}=\sigma_{i}^{2}\left(P^{\top} \Psi\right)$. It is easy to sce that $\sigma_{i}^{2}(\Psi)=$ $1 \pm|\phi|$. This corresponds to $\lambda_{i}$ for the special case $\xi=0$, i.e., at the vertical axis, with $P$ being identity, the temporally uncorrelated equal-powered arrival case. We also have

$$
\sigma_{i}^{2}(P)=\frac{1}{2}\left(\frac{\pi_{1}}{\pi_{2}}+1\right)\left[1 \pm \sqrt{1-\frac{1\left(\frac{\pi_{1}}{\pi_{2}}\right)\left(1-|\rho|^{2}\right)}{\left(\frac{\pi_{1}}{\pi_{3}}+1\right)^{2}}}\right] .
$$

'This corresponds to $\lambda_{i}$ for the special case $\xi=0$, i.c., at the vertical uxis, with $\Psi$ bcing identity, the sputially orthogomal arrival case.

Given these algebraic expressions, we now interpret their physical significance. The thrce main sources for the signal eigenvalue spread or causes for small signal eigenvalucs are the high spatial correlation $\phi$ due to narrow spatial separation between two arrivals, the high temporal corrclation $\rho$ because of multipaths, and the high strength contrast $\frac{\pi_{1}}{\pi_{2}}$. We note that $\phi$ appears in $\Psi, \rho$ and $\frac{\pi_{1}}{x_{2}}$ in $P$. Speiscr's eigenvalue bounds for the product of two matrices tell us that if we know the cigenvalues of $\Psi$ and $P$, then we can bound the eigenvalues, $\lambda_{2}=\sigma_{2}^{2}(P \Psi)$, of the product matrix. Stated loosely, if we know the partial stress from spatial correlation alone and that from temporal correlation together with the power contrast, we can bound the joint stress from all of the above combined sources. From the above expressions, we know that the partial stresses $\sigma_{2}^{2}(\Psi)$ and $\sigma_{2}^{2}(P)$ depend on $\phi$ and $\rho$ constrained on the two unit-disks only through their mugniludes, i.c., radii, but nou the angular positions. Yet the combined stresses $\lambda_{2}=\sigma_{2}^{2}(P \Psi)$ depend also on the relative phasor positions. Recognizing the nature of the many-to-one mapping from $(\rho, \phi)$ to $(\xi, \eta)$ which appeared in the eigenvalue formulas, we note that the rclative phasor positions appeared only in the phase-dependant variable $\xi$.

For given $|\rho|$ and $|\phi|$, we have $\eta=\left(1-|\phi|^{2}\right)\left(1-|\rho|^{2}\right)$ and $|\xi|_{\max }=|\rho||\phi|$. It is of interest to see whether Speiser's uprer and lower bounds for $\lambda_{2}$ coincide with

$\lambda_{2}\left(-|\rho||\phi|,\left(1-|\phi|^{2}\right)\left(1-|\rho|^{2}\right)\right)$ and

$\lambda_{2}\left(|\rho||\phi|,\left(1-|\phi|^{2}\right)\left(1-|\rho|^{2}\right)\right)$. We also like to know whether these bounds are tight or loose as the scenario points range over the isosceles region for the equipowered arrival $\frac{\pi_{1}}{\pi_{2}}=1$ case and the large power-ratio case.

For the Special Finuipower Case $\frac{\pi_{1}}{x_{2}}=1$

$$
\text { For } \begin{aligned}
\frac{\pi_{1}}{\pi_{2}}= & 1 \text {, Speiser's expreson states that } \\
& (1-|\rho|)\left(1-\mid \phi^{\prime}\right) \leq \lambda_{2} \\
\leq & \min [(1+|\phi|)(1-|\rho|),(1-|\phi|)(1+|\rho|)] \\
= & (1-|\rho||\phi|)-|| \Gamma_{1}^{\prime}-|\phi| \mid .
\end{aligned}
$$

These bounds have exactly the same expressions for $\lambda_{2}$ at the end points of the permissible $\xi$-interval between $\left(-|\rho||\phi|,\left(1-|\phi|^{2}\right)\left(1-|\rho|^{2}\right)\right)$ and $\left(|\rho||\phi|,\left(1-|\phi|^{2}\right)\left(1-|\rho|^{2}\right)\right)$ when $\frac{\pi_{1}}{\pi_{2}}=1$ as shown in the following.

For a given $\eta$ value and $\frac{\pi_{1}}{\pi_{2}}=1$, as $\xi$ varies from the left edge of tise permissible $\xi$-interval to the midpoint and then to the right edge, $\lambda_{2}$ decreases from

$$
\begin{aligned}
& \lambda_{2}\left(-|\rho||\phi|,\left(1-|\phi|^{2}\right)\left(1-|\rho|^{2}\right)\right) \\
= & (1-|\rho||\phi|)-\sqrt{(1-|\rho||\phi|)^{2}-\left(1-|\phi|^{2}\right)\left(1-|\rho|^{2}\right)} \\
= & (1-|\rho||\phi|)-|| \rho|-| \phi||,
\end{aligned}
$$


to

$$
\lambda_{2}\left(0,\left(1-|\phi|^{2}\right)\left(1-|\rho|^{2}\right)\right)=1-\sqrt{1-\left(1-|\phi|^{2}\right)\left(1-|\rho|^{2}\right)}
$$

to

$$
\begin{aligned}
& \lambda_{2}\left(|\rho||\phi|,\left(1-|\phi|^{2}\right)\left(1-|\rho|^{2}\right)\right) \\
= & (1+|\rho||\phi|)-\sqrt{(1+|\rho||\phi|)^{2}-\left(1-|\phi|^{2}\right)\left(1-|\rho|^{2}\right)} \\
= & (1+|\rho||\phi|)-(|\rho|+|\phi|)=(1-|\phi|)(1-|\rho|) .
\end{aligned}
$$

So for $\frac{\pi_{1}}{\pi_{2}}=1$, Speiser's two bounds for the equipower case can be identified as the eigenvalue values at the two extreme ends of the permissible phase-dependent variable $\xi$-interval. This $\xi$-interval is on the constant phaseindependent $\eta$ horizonta! line of the isosceles region with all expressions calculated from the magnitudes of $\rho$ and $\phi$ only. For given $\eta$, the permissible $\xi$-interval at tains its maximum width when the $\xi$-interval extends from the left- to the right-boundary parabola. The leagth of such maximum $\xi$ interval increases with decreasing $\eta$, i.e., when the baseline is approached. The hounds for the small eigenvalue may be loose when there is considerable variation of the order of magnitule of the small cigenvalue along the maximum $\xi$-interval.

\section{For the General Power Ratio $\frac{\pi_{1}}{\pi_{2}}$ Case}

For the general $\frac{\pi_{1}}{\pi_{2}}$ case, Speiser's expression can be similarly arranged as $\hat{N}_{0} .0 \mathrm{~A} 6 \mathrm{~mm}$

$$
\begin{aligned}
(1- & |\phi|) \frac{1}{2}\left(\frac{\pi_{1}}{\pi_{2}}+1\right)\left[1-\sqrt{1-\frac{4\left(\frac{\pi_{1}}{\pi_{2}}\right)\left(1-|\rho|^{2}\right)}{\left(\frac{\pi_{1}}{\pi_{2}}+1\right)^{2}}}\right] \leq \lambda_{2} \\
\leq & \frac{1}{2}\left(\frac{\pi_{1}}{\pi_{2}}+1\right)\left\{1-|\phi| \sqrt{1-\frac{4\left(\frac{\pi_{1}}{\pi_{2}}\right)\left(1-|\rho|^{2}\right)}{\left(\frac{\pi_{1}}{\pi_{2}}+1\right)^{2}}}-\right. \\
& \left.|| \phi\left|-\sqrt{1-\frac{1\left(\frac{\pi_{1}}{\pi_{2}}\right)\left(1-|\rho|^{2}\right)}{\left(\frac{\pi_{1}}{\pi_{2}}+1\right)^{2}}}\right|\right\} .
\end{aligned}
$$

Note these bound expressions involve only the absolute magnitude of the two phasors on the two unit-disks, i.e., only the radius but not the argument of the phasors. They are not explicitly dependent on the of her parameter

$$
\xi=\operatorname{Re}\left(\rho \phi^{*}\right)=|\rho||\phi| \cos (\arg \rho-\arg \phi),
$$

which is the inner product of these two phasors and consequently contains relative phase information between the two. But as before, for given $|\rho|$ and $|\phi|$, we have $\eta=\left(1-|\phi|^{2}\right)\left(1-|\rho|^{2}\right)$ and $|\xi|_{\max }=|\rho||\phi|$. Therefore, as in the preceding subsection for the equipower casc $\frac{x_{1}}{\pi_{2}}=1$, we can draw the conclusion a priori that these bound expressions at best bound the lower part of the truncated hyperbola in $\xi$ and $\lambda$ over the permissible phase-dependent variable $\xi$-interval corresponding to the given value of

$$
\eta=\left(1-|\phi|^{2}\right)\left(1-|\rho|^{2}\right) \text {. }
$$

For given value of $\frac{\pi_{1}}{\pi_{2}}$, the exnet cigenvalues depend on the two complex pliasors on the unit-disks, $\rho$ and $\phi$, through the two derived bounded real numbers $\xi$ and $\eta$. In contrast, the two bounds' expressions involve the magnitude of the two phasors, i.e., two different real numbers. Therefore, it is much simpler to browse the hyperbolic slice plots to visualize the best non-explicitly $\xi$-dependent bounds one can possibly have than to analyze the particular bounds derived hy Speiser. Frum unr early characterization of the $\lambda$ surfares, we know that. the: dependence of $\lambda s$ on $\xi$ becomes more insignificant as $\frac{\pi_{1}}{\pi_{2}}$ gets large. We expect that such $\xi$-independent bounds perform better in this region. Naturally we must bear in mind that the original form of these bounds are applicable to the general multisource arrival sceunrio and llicy muy serve some useful purpose there.

\section{Conclusion}

The main contribution of this work is a manageable presentation of a compact map showing the three functions, $\lambda_{1}, \lambda_{2}$, and $\frac{\lambda_{1}}{\lambda_{2}}$, over all possible scenarios. This enables one to see the relative positions among different scenarios. We also present some easy-to-rencmber formulas that cnable one to exercise back-of-envelope assessment of scenarios.

We also characterize Speiser's eigenvalue bounds specialized to the two source scenario. These bound expressions involve only the absolute magnitude of the two phasors on the two unit disks, i.c., only the radius but not the argument. of the phasors. Interestingly enough, his two bounds for the equi-power case can be identified as the eigenvalue values it the two extreme ends of the permissible phase-dependent variable $\xi$-interval on the constant phase-independent $\eta$ horizontal line of the isosceles region with all expressions calculated from the magnitudes of $\rho$ and $\phi$ only. For given $\eta$, the permissible $\xi$-interval attains its maximum width when the $\xi$-interval extends from the left to the right boundary parabola. The length of such maximum $\xi$-interval increases with decreasing $\eta$, i.e., when the bascline is npproaclied. The boumds for the small eigenvalue muy be loose when there is considerable variation in the order of magnitude of the small eigenvalue in the phase-dependent variable $\xi$. For large $\frac{\pi_{1}}{\pi_{3}}$, the smaller eigenvalue $\lambda_{2}$ becomes more independent of the horizontal phase-dependent variable $\xi$, the bounds will get tigliter. That is, there is less information loss by neglecting the phase-dependent variable $\xi$.

\section{References}

[1] Iludson, J.E., Adaptive Army Principles, Peter Peregrinus I,td., I,ondon, 1981.

(2) Chou, S.I., Eigenvalues of Covariance Matrix for TwoSource Array Processing, in Proc. $24^{\text {th }}$ Asilomar Conf. Sig., Syst., Compt., Pncific Grove, Californin, November $5-7,1990$.

[3] Speiser, J., and Arnold, II.B., Array Manifold Effects in Subspace Techniques, in Proc. of the SPIE: Advanced Algorithms \& Architectures for Signal Processing IV, SPIE Vol. 1152, Dec. 1989.

[1] Chou, S.I., Eigenvalues of Covariance Mfatrix for TwoSource Array Processing, NOSC TR 1370, Naval Ocean Systems Center, San Diego, CA, Oct 1990. 